\title{
Reptiles et Amphibiens de l'Inde méridionale.
}

\author{
par \\ Jean ROUX
}

Musée d'Histoire naturelle de Bâle.

La collection erpétologique qui fait l'objet de la présente étude a été formée par MM. les Drs J. CarL (Genève) et K. Escher (Zurich) au cours de leur voyage scientifique dans l'Inde méridionale, en 1926-1927; elle comprend 60 espèces.

Il faut rappeler ici que l'exploration de ces deux naturalistes était plus spécialement dirigée vers la faune des Invertébrés, c'est ce qui explique le nombre relativement faible des espèces collectionnées; il ne représente pas même un quart de la riche faune erpétologique du Sud de l'Inde qui comprend environ 260 espèces.

La présente collection ne renferme pas d'espèces nouvelles, les Reptiles et Amphibiens de cette région étant déjà fort bien connus; nous avons seulement décrit comme sous-espèce nouvelle, un Agamidé représenté par un seul individu, différant quelque peu de Salea anamallayana typique.

Nous avons pris comme base de notre étude la belle monographie de Boulenger ${ }^{1}$, parue en 1890, mais nous avons été obligé — bien à regret - de modifier la dénomination de plusieurs espèces, suivant en cela l'exemple de WALL. Depuis la publication du volume de Boulenger, nombre de travaux erpétologiques ont paru dans diverses revues scientifiques, en particulier dans les Records of Indian Museum de Calcutta et dans le Journal of the Bombay

1 Boulenger G.A. Reptilia and Batrachia in : Fauna of British India, including Ceylon and Burma. London 1890.

Rev. Suisse de Zool., T. 35. 1928. 
Natural History Society. Dans ce dernier périodique, $\mathrm{W}_{\mathrm{ALL}}{ }^{1}$ a publié, en 1923 et 1924, une liste des serpents de l'Inde, avec indications de leur répartition géographique et de la bibliographie, ce qui nous dispensera de répéter, pour les Ophidiens, ces données relatives à la période allant de 1890 à 1923-24.

Nous tenons à remercier M. le $\mathrm{D}^{\mathrm{r}} \mathrm{J}$. CARL d'avoir bien voulu nous confier les matériaux, fort bien conservés, qu'il a rapportés, et M. le $\mathrm{D}^{\mathrm{r}}$ Fr. Sarasin, qui nous a autorisé à profiter des richesses de sa bibliothèque.

En énumérant les espèces, nous ajoutons, quand cela nous a semblé utile, quelques renseignements sur leur morphologie ou leur coloration. Les provenances ont été soigneusement notées, avec l'altitude des diverses localités. En terminant, nous donnerons un court aperçu de l'histoire du peuplement erpétologique du Sud de l'Inde, d'après l'étude de M. le Dr Fr. Sarasin ${ }^{2}$, parue en 1910.

\section{REPTILIA}

\section{OPHIDIA}

\section{Famille Typhlopidæ.}

\section{Typhlops braminus (Daud.)}

1890. Boulenger, G. A. Fauna of Brit. India, Rept. et Batr. p. 236. 1923. Wall, F. Journal Bombay Nat. Hist. Soc., vol. 29, p. 349.

Localité: Coimbatore, plaine, 400 m., 11-13.XII.1926. 3 Ex.

Ces exemplaires concordent parfaitement avec la description de Boulenger. Les écailles sont tachées de noir à leur bord antérieur.

Cette espèce a une énorme répartition géographique; on la trouve de l'Afrique centrale à l'Archipel indo-australien et elle habite également Madagascar.

1 Wall, F. A Hand-list of the Snakes of the Indian Empire, in Journal Bombay Natur. History Society, Vol. 29, 1923, Part. 1, p. 345-361, Part. 2, p. 598-632; 1924, Part. 3, p. 864-878; Vol. 30, 1924, Part. 4, p. 12-24; 1925, Part. 5, p. 242-252.

2 Sarasin Fr. Ueber die Geschichte der Tierwelt von Ceylon. Zool. Jahrbücher, Abt. f. Systematik, Suppl. XII, Heft. 1. 1910. 


\section{Typhlops beddomei Blgr.}

1890. Boulenger, G. A. Fauna of Brit. India, Rept. et Batr., p. 237. 1923. Wall, F. Journ. Bombay Nat. Hist. Soc., vol. 29, p. 348.

Localité: Tandikudi, Palnis, 1500 m., 23.IV.1927, 2 Ex.

L'un de ces exemplaires a une longueur de $125 \mathrm{~mm}$., l'autre de $110 \mathrm{~mm}$. Les plaques antérieures de la tête sont de couleur claire et pourvues de fines réticulations brunes; le corps est brun, plus pâle en dessous qu'en dessus. Pas de strie dorsale plus foncée; région anale blanche. On compte 10 écailles subcaudales.

Cette espèce est répandue dans la péninsule, à partir du Sud de la plaine du Gange.

\section{Famille Uropeltidæ.}

\section{Silybura pulneyensis (Bedd.)}

1890. Boulenger, G. A. Fauna of Brit. India, Rept. et Batr., p. 260. 1923. Wall, F. Journ. Bombay Nat. Hist. Soc., vol. 29, p. 356 et 392.

Localités: Kukkal, Palnis, 1900 m., 2.IV.1927, 1 Ex. ô. Pumbarai, Palnis, 2000 m., 25.III.1927, 1 Ex. Mariyanshola, Palnis, 2500 m., 12.IV.1927.1 Ex.

C'est ce dernier individu qui est le plus grand, il mesure $245 \mathrm{~mm}$ de longueur. On compte 154 ventrales et 8 subcaudales. Chez l'exemplaire de Kukkal (174 ventrales, 12 subcaudales) les taches ventrales jaunes sont moins nombreuses que chez les autres spécimens, surtout dans la partie moyenne et postérieure.

\section{Silybura broughami Bedd.}

1890. Boulenger, G. A. Fauna of Brit. India, Rept. et Batr., p. 264. 1923. Wall, F. Journ. Bombay Nat. Hist. Soc., vol. 29, p. 359.

Localité: Tandikudi, Palnis, 1500 m., 23.IV.1927, 6 Ex.

Le plus grand de ces exemplaires a une longueur totale de $280 \mathrm{~mm}$. On compte 181 ventrales et 11 subcaudales. Cette espèce est endémique et n'est connue que des Palnis.

\section{Silybura macrorhyncha Bedd.}

1890. Boulenger, G. A. Fauna of Brit. India, Rept. et Batr., p. 264. 1923. Wall, F. Journ. Bombay Nat. Hist. Soc., vol. 29, p. 357.

Localité: Attakatti, Anaimalais, 1000 m., 3.III.1927, 1 Ex. Le diamètre de cet individu, dont la longueur totale est de 
$350 \mathrm{~mm}$, est compris 35 fois dans cette dernière dimension. On compte 203 ventrales. La coloration est conforme à celle qu'indique BOULENGER.

Cette espèce est endémique et n'est connue que des Palnis.

\section{Plecturus perroteti (D.B.).}

1890. Boulenger, G. A. Fauna of Brit. India, Rept. et Batr., p. 271. 1923. Wall, F. Journ. Bombay Nat. Hist. Soc., vol. 29, p. 359.

Localités: Coonoor, Nilgiris, 1850 m., I.1927, 10 Ex. Avalanche, Nilgiris, 2100 m., 18.I.1927, 1 Ex. sans étiquette, 1 Ex.

Le plus grand de ces spécimens mesure $340 \mathrm{~mm}$; sa couleur générale est brune, les écailles ayant leur centre plus clair que les bords. D'autres individus sont d'un brun uniforme sur le dos, plus pâles en dessous; la région anale est jaunâtre. On remarque parfois une strie jaunâtre, médiane, sur la partie supérieure de la queue.

Cette espèce est particulière au Sud de l'Inde.

\section{Platyplecturus madurensis Bedd.}

1890. Boulenger, G. A. Fauna of Brit. India, Rept. et Batr., p. 274. 1902. Ferguson, H. S. Journ. Bombay Nat. Hist. Soc., vol. 14, p. 387.

1923. Wall, F. Journ. Bombay Nat. Hist. Soc., vol. 29, p. 360 et 396.

Localité: Maryland, Palnis, 1600 m., 21.IV.1927, 1 Ex.

Cet exemplaire a une longueur totale de $305 \mathrm{~mm}$. Les plaques ventrales sont au nombre de 166, les subcaudales de 9. L'animal est brun en dessus; les écailles de la face ventrale sont plus claires et bordées de brun. Le diamètre du corps entre 38 fois dans la longueur totale.

Ferguson mentionne cette espèce pour les Cardamom Hills (Travancore) à environ 3000 pieds d'altitude. C'est une espèce endémique.

\section{Famille Colubridæ.}

\section{Xylophis perroteti (D.B.)}

1890. Boulenger, G. A. Fauna of Brit. India, Rept. et Batr., p. 283. 1895. Ferguson, H. S. Journ. Bombay Nat. Hist. Soc., vol. 10, p. 71. 1923. Wall, F. Journ. Bombay Nat. Hist. Soc., vol. 29, p. 398 et 610. 
Localités: Coonoor, Nilgiris, 1850 m., XII.1926-I.1927, 7 Ex. Valparai, Anaimalais, 1100 m., 10.III.1927, 1 Ex. Maryland, Palnis, 1600 m., 18.IV.1927, 1 Ex.

Le plus grand des spécimens de Coonoor mesure environ $400 \mathrm{~mm}$; il possède 145 ventrales et 25 subcaudales. Ces individus sont d'un gris noirâtre sur le dos; en dessous les écailles sont d'un gris clair, bordées de noir. La région gulaire est blanchâtre, avec une raie médiane, longitudinale, de couleur grise, qui s'élargit d'avant en arrière.

L'exemplaire de Maryland est d'un brun rougeâtre, avec de nombreuses taches noires, disposées en séries longitudinales plus ou moins irrégulières. Les ventrales sont d'un brun jaune avec macules noirs; la raie ventrale médio-antérieure est bien marquée.

Cette espèce est particulière au Sud de l'Inde.

\section{Lycodon travancoricus Bedd.}

1890. Boulenger, G. A. Fauna of Brit. India, Rept. et Batr., p. 293. 1923. Ophites travancoricus, Wall, F. Journ. Bombay Nat. Hist. Soc., vol. 29, p. 613.

1924. Lycodon travancoricus, Wall, F. Journ. Bombay Nat. Hist. Soc., vol. 30, p. 252.

Localités: Coonor, Nilgiris, 1900 m., XII.1926, 5 Ex. juv. Maryland, Palnis, 1600 m., 19.IV.1927, 1 Ex.

Les exemplaires jeunes sont noirâtres, avec bandes transversales blanches bien accusées, ainsi que le dessin latéral en zig-zag. Chez l'individu plus grand, provenant de Maryland, le fond est brun et les taches transversales ont presque disparu. Il possède 188 ventrales, la queue est mutilée.

Cette espèce ne se rencontre que dans le Sud de l'Inde; elle est commune, soit dans la plaine, soit sur les collines.

\section{Ptyas mucosus (L.).}

1890. Zamenis mucosus. Boulenger, G. A. Fauna Brit. India, Rept. et Batr., p. 324.

1914. Zaocys mucosus Wall, F. Journ. Bombay, Nat. Hist. Soc. vol. 23, p. 168.

1923. Zaocys mucosus. Hora, S. L. et Chopra, B. Records Ind. - Museum, vol. XXV, p. 375.

1923. Ptyas mucosus. Wall, F. Journ. Bombay Nat. Hist Soc., vol. 29 , p. 617. 
Localité: Kukkal, Palnis, 1900 m., 2.IV.1927, 1 Ex.

Cet individu, dont la longueur totale est de 2 mètres, a été capturé dans des champs d'oignons.

Dans son travail de 1914, WALL a transféré tout d'abord cette espèce du genre Zamenis dans le genre Zaocys. Sa répartition géographique s'étend sur toute l'Asie méridionale. C'est un des serpents les plus communs, en plaine comme en montagne.

\section{Coluber helena Daud.}

1890. Boulenger, G. A. Fauna Brit India, Rept. et Batr. p. 331. 1913. Wall, F. Journ. Bombay Nat. Hist, Soc., vol. 22, p. 22, pl. XIX. 1923. Wall, F. Journ. Bombay Nat. Hist. Soc.,vol. 29, p. 622.

Localités: Coonoor, Nilgiris, 1850 m., 10.I.1927, 1 Ex. juv. Attakatti, Anaimalais, 1000 m., 24.II.1927, 1 Ex.juv.

Ces deux individus ont encore, très distincte, la livrée du jeune. Cette espèce, commune dans diverses régions de l'Inde et à Ceylan, semble être surtout répandue dans les régions de collines et dans les montagnes. Dans son travail de 1913 cité ci-dessus, WaLL donne une carte de sa distribution dans l'Inde et Ceylan.

\section{Rhabdophis beddomei (Gthr.).}

1890. Tropidonotus beddomii. Boulenger, G. A. Fauna Brit. India, Rept. et Batr., p. 344.

1895. Tropidonotus beddomii. Ferguson, H. S. Journ. Bombay Nat. Hist. Soc., vol. 10, p. 72.

1923. Rhabdophis beddomei WaLL, F. Journ. Bombay Nat. Hist. Soc., vol. 29 , p. 605 .

Localités: Coonoor, Nilgiris, 1850 m., 2.I.1927, 5 Ex.

Attakatti, Anaimalais, 1000 m., 28.II.1927, 1 Ex.

Le plus grand spécimen a une longueur totale de $370 \mathrm{~mm}$; il possède 149 ventrales et 73 subcaudales.

Chez quelques individus la face dorsale est tachetée de noir et de blanc; le collier nuchal est visible chez les plus jeunes exemplaires, il peut s'élargir en envahissant la partie postérieure de la tête. Les ventrales sont claires, avec de petites taches brunes latérales.

Cette espèce est spéciale aux régions montagneuses du Sud de l'Inde, où d'après Ferguson elle est commune, à partir de 1500 pieds d'altitude. 


\section{Rhabdophis monticola (Jerd.).}

1890. Tropidonotus monticola. Boulenger, G. A. Fauna Brit. India, Rept. et Batr., p. 347.

1923. Rhabdophis monticola. Wall, F. Journ. Bombay Nat. Hist. Soc., vol. 29 , p. 607.

Localité: Mudumalai, Nilgiris, 1000 m., 8.II.1927, 1 Ex. Cet individu possède 142 ventrales et 88 subcaudales. Il est d'un gris olivâtre sur le dos, avec des bandes transversales plus foncées, visibles surtout dans la moitié postérieure du corps. Ces taches, un peu plus larges sur le dos que sur les côtés, sont bordées d'une fine ligne de teinte plus claire. La ligne blanche arquée, qui orne le dessus de la tête en arrière de la frontale, est bien visible. Région périoculaire blanchâtre ainsi que les supralabiales médianes qui présentent aussi quelques macules noirs.

Cette espèce est confinée dans les régions montagneuses du Sud-Ouest de l'Inde.

\section{Rhabdophis stolatus (L.).}

1890. Tropidonotus stolatus. Boulenger, G. A. Fauna Brit. India, Rept., et Batr., p. 348.

1923. Rhabdophis stolatus. WALL, F. Journ. Bombay Nat. Hist. Soc., vol. 29, p. 605.

Localités: Kodaikanal-Road, en plaine, 200 m., 25.III.1927, 1 Ex. Kukkal, Palnis, 1900 m., IV.1927, 1 Ex.

L'exemplaire capturé en plaine est d'un brun olivâtre, celui de Kukkal d'un brun plus franc, les taches transversales noires bien visibles et traversées par les deux lignes longitudinales claires qui sont d'une teinte légèrement jaunâtre. Espèce commune en Inde, dans les régions du Sud-Est du continent asiatique et dans l'Archipel malais.

\section{Nerodia piscator (Schn.).}

1890. Tropidonotus piscator. Boulenger, G. A. Fauna Brit. India Rept. et Batr., p. 349.

1907. Tropidonotus piscator. WALL, F. Journ. Bombay Nat. Hist. Soc., vol. 17, p. 857, pl. IV.

Nerodia piscator. WAll, F. Journ. Bombay Nat. Hist. Soc., vol. 29 , p. 603.

Localité: Valparai, Anaimalais, 1100 m.,7.III.1927, 1 Ex. 
Cet exemplaire possède 140 ventrales et 48 subcaudales. Sa couleur générale est d'un gris de plomb avec petites taches noires peu apparentes. Le ventre est blanc, sans taches; les plaques sont bordées latéralement de gris.

Espèce aquatique dont la livrée est très variable, qu'on rencontre communément dans toute la région orientale.

\section{Macropisthodon plumbicolor (Cant.)}

1890. Tropidonotus plumbicolor. Boulenger, G. A., Faun. Brit. India, Rept. et Batr., p. 351.

1893. Macropisthodon plumbicolor. Boulenger, G. A. Cat. Snakes Br. Mus. Vol. I, p. 267.

1907. Macropisthodon plumbicolor. Wall, F. Journ. Bombay Nat. Hist. Soc., vol. 17, p. 1, pl. II.

1923. Macropisthodon plumbicolor. Wall, F. Journ. Bombay Nat. Hist. Soc., vol. 29, p. 607.

Localité: Coonoor, Nilgiris, 1850 m., 17.I.1927, 1 Ex.

Cet exemplaire a $300 \mathrm{~mm}$ de long, il possède 150 ventrales et 43 subcaudales. On compte 23 écailles autour de la partie médiane du corps. Il est d'un vert sombre en dessus, avec de rares taches noires dans la moitié antérieure du dos; ces taches sont disposées en bandes transversales. Ventre d'un gris uniforme. Espèce répandue dans l'Inde et à Ceylan; elle est très commune dans les districts méridionaux de la péninsule.

\section{Helicops schistosus (Daud.)}

1890. Boulenger, G. A. Fauna Brit. India, Rept. et Batr., p. 352. 1923. Wall, F. Journ. Bombay Nat. Hist. Soc., vol. 29, p. 608.

Localité: Kukkal, Palnis, 1900 m., 3.IV.1927, 1 Ex.

Ce spécimen, récolté dans une rizière inondée, possède 139 ventrales et 79 subcaudales. Il est d'une couleur grise, avec de petites taches noires, visibles surtout sur les côtés. WaLL dit que c'est surtout une espèce de plaine, mais qui habite aussi le plateau de Mysore et qui a été également signalée dans les montagnes d'Anaimalais et de Wynaad.

Espèce qu'on rencontre dans le voisinage des eaux dans toute la région du Sud-Est de l'Asie. 


\section{Dryophis dispar (Gthr.)}

1890. Boulenger, G. A. Fauna Brit. India, Rept. et Batr., p. 352.

1907. Mocquard, F. Les Reptiles de l'Indo-Chine, in: Revue Coloniale, Paris, p. 49.

1924. Wall, F. Journ. Bombay Nat. Hist. Soc., vol. 29, p. 877.

Localités: Tanakka-Malay, Anaimalais, 2400 m., 10.III.1927, 4 Ex.

Pumbarai, Palnis, 2000 m., 27.III.1927, 1 Ex.

Mariyanshola, Palnis, 2500 m., 14.IV. 1927, 1 Ex.

Chez le grand exemplaire de Tanakka Malay, on compte 138 ventrales et 105 subcaudales.

Dans quelques cas, les internasales et préfrontales sont en contact avec les labiales supérieures, ailleurs il y a séparation par les loréales, au nombre de 1 à 3 , généralement 2 .

Cette espèce semble assez commune sur les collines du Sud de l'Inde, elle a été signalée dans l'Indo-Chine par Mocquard, mais WaLl pense qu'il y a erreur, soit de détermination, soit de provenance.

\section{Naja naja (L.)}

1890. Naia tripudiars. Boulenger, G. A. Fauna Brit. India, Rept. et Batr., p. 391, fig. 115.

1925. Naia naia (L.). Wall, F. Journ. Bombay Nat. Hist. Soc., vol. 30, p. 242 .

Localité: Masinigudi, Nilgiris, 1000 m., 1 Ex.

Espèce très commune dans toute la partie sud-orientale de l'Asie.

\section{Famille Viperidæ.}

\section{Ancistrodon millardi Wall.}

1908. Wall, F. Journ. Bombay Nat. Hist. Soc., vol. 18, p. 792.

1925. Wall, F. Journ. Bombay Nat. Hist. Soc., vol. 30, p. 249.

Localité: Attakatti, Anaimalais, 1000 m., 3.III.1927, 1 q grav.

Après l'examen d'un nombreux matériel, WALL a séparé cette espèce de $A$. hypnale (Merr.), dont elle est fort voisine. Elle en diffère entre autres par le nombre plus grand des plaques ventrales (136-152 au lieu de 116-131) et des subcaudales (30-44 au lieu de 24-37). Notre sujet possède 140 ventrales et 40 subcaudales; il 
est d'un gris brun sur le dos, avec 2 séries longitudinales de taches noires sur la région médio-dorsale, où elles sont disposées par paires. Les côtés de la tête sont bruns et ourlés en dessus d'une fine ligne claire qui se continue sur les côtés du cou. Sur les écailles latérales de la dernière série, quelques taches noires forment une rangée longitudinale complète.

Cette espèce habite les montagnes du Sud-Ouest de l'Inde et se rencontre également dans les contrées montagneuses de Ceylan.

\section{Trimesurus anamallensis (Gthr.)}

1890. Boulenger, G. A. Fauna of Brit. India, Rept. et Batr., p. 430. 1925. Wall, F. Journ. Bombay Nat. Hist. Soc., vol. 30, p. 250.

Localité: Coonoor, Nilgiris, 1850 m., 28.XII.1926, 1 Ex.

On compte chez cet individu 21 séries d'écailles autour du corps; il possède 148 ventrales et 47 subcaudales. De couleur verte, il est marbré de noir sur le dos; sa queue est jaune et noire et la face ventrale d'un vert clair.

D'après WaLl, cette espèce est confinée dans les régions montagneuses de l'Inde méridionale, au Sud de la Rivière Krishna. Elle est commune aux altitudes allant de 2000 à 7000 pieds.

\section{LACERTILIA}

\section{Famille Geckonidæ.}

\section{Gonatodes indicus (Gray).}

1890. Boulenger, G. A. Fauna of Brit. India, Rept. et Batr., p. 74. 1905. Annandale, N. L. Journ. Asiat. Soc. Bengal, vol. 1, p. 91.

Localités: Bangitappali, Nilgiris, 2400 m.,20. I.1927, 4 ô juv., 4 + juv.

Ootacamund, Nilgiris, 2250 m., 12.I.1927, 2 ô.

Cette espèce appartient au groupe dont le corps est dépourvu, sur les flancs, de tubercules spiniformes saillants. Tandis que les spécimens de Bangitappai ont les écailles ventrales lisses, ceux d'Ootacamund les ont légèrement carénées. Le corps est d'un brun plus 
ou moins foncé, avec marbrures plus sombres; souvent on observe une fine ligne claire, médio-dorsale, plus ou moins longue. Espèce spéciale au Sud de l'Inde.

\section{Gonatodes gracilis (Bedd.).}

1890. Boulenger, G. A. Fauna of Brit. India, Rept. et Batr., p. 78. 1905. Annandale, N. L. Journ. Asiat. Soc. Bengal, vol. 1, p. 91. 1926. Hora, S. L. Records Ind. Mus., vol. 28, p. 191, pl. VII, fig. 7.

Localité: Attakatti, Anaimalais, 1000 m., 25.II.1927, 4 ô, 1 ㅇ juv.

D'après Boulenger cette espèce se distinguerait de la forme voisine G. kandianus (Kel.) par l'absence de carènes sur les ventrales. Mais, comme Hora le fait remarquer et ainsi que j'ai pu le constater moi-même sur divers exemplaires de Ceylan, G. kandianus présente, à cet égard, de grandes variations. Hor indique comme caractère distinctif entre les deux espèces, la forme de la mentale et l'arrangement des plaques qui la suivent immédiatement en arrière (voir sa fig. du texte 3 ). Alors que chez kandianus on trouverait 3 plaques en arrière de la mentonnière, il n'y en aurait que 2 formant une suture médiane chez gracilis. Ce caractère n'est pas constant non plus, ainsi que nous l'avons constaté chez ces individus dont deux seulement sur les cinq présentent la suture médiane des 2 plaques. Peut-être pourrait-on distinguer ces deux espèces par le nombre des lamelles sous le $4^{\mathrm{me}}$ orteil. Tandis que ce nombre ne varie que de 6 à 7 pour le segment basal chez les deux espèces, il est, au segment terminal, de 11 à $12 \mathrm{chez}$ les individus ceylanais de kandianus que nous avons examinés et de 13 à 15 chez gracilis. La queue diffère aussi par sa coloration et par la conformation des tubercules carénés qu'elle porte; ceux-ci font plus fortement saillie et sont plus larges à leur base chez gracilis que chez kandianus.

La coloration de nos spécimens concorde avec celle qu'indique Boulenger. Ils sont de teinte grise et ornés de taches plus claires, arrondies, dont les médio-dorsales sont les plus grandes. La nuque porte une tache noire et des lignes foncées rayonnent en arrière de l'œil. Des taches foncées se voient sur les labiales et des lignes Iongitudinales noirâtres sur les côtés du cou. La queue est annelée, en dessus, de noir et de blanc et sa partie inférieure est d'un blanc 
grisâtre. L'un des jeunes exemplaires est de couleur générale plus foncée que les autres.

D'après Hora, le trone et les membres peuvent être aussi ornés de bandes transversales noires et blanches semblables à celles de la queue.

Cette espèce, selon Boulenger, habite le Sud de l'Inde et Ceylan.

\section{Gonatodes jerdoni Theob.}

1890. Boulenger, G. A. Fauna of Brit. India, Rept. et Batr., p. 78. 1905. Annandale, N. L. Journ. Asiat. Soc. Bengal, vol. 1, p. 91. 1922. Wall, F. Journ. Bombay Nat. Hist. Soc., vol. 28, p. 494.

1926. Hora, S. L. Records Ind. Mus., vol. 28, p. 192.

Localités: Coonoor, Nilgiris, 1850 m., XII,1926, 3 ô 1 ․ Attakatti, Anamalais, 1000 m., 6.II.1927, 1 ô juv. Tandikudi, Palnis, 1500 m., 23.IV.1927, 1 ô juv.

Les exemplaires répondent bien à la description de Boulenger. L'écaillure du dos est uniforme; sur les flancs quelques écailles plus grandes font saillie. Les écailles de la queue sont lisses; dans sa partie basale on remarque quelques tubercules plus larges. Chez cette espèce, les orteils possèdent sur leur face inférieure de larges plaques dans la partie basale; on en compte 5 dont la distale est la plus grande; sous le segment suivant ces plaques sont plus étroites et au nombre de 10 à 11 .

Les individus de Coonoor sont d'un gris brun en dessus, avec lignes transversales ondulées, plus foncées, arquées en avant et dont la portion médiane est la mieux indiquée. Sur la ligne médiodorsale, ces bandes foncées sont suivies d'une tache claire. Les labiales sont tachées de brun foncé. Sur la nuque une tache en W, une barre transversale foncée en avant de l'œil et quelques lignes foncées sur les côtés de la tête. Chez le $\widehat{o}$ la gorge a une teinte grisâtre et les pores fémoraux sont au nombre de 5 à 6 .

Selon Boulenger, cette espèce habite le Sud de l'Inde et l'île de Ceylan.

\section{Hemidactylus frenatus (D.B.).}

1890. Boulenger, G. A. Catal. of Brit. India, Rept. et Batr., p. 85. 1905. Annandale, N. L. Journ. Asiat. Soc. Bengal, vol. 1, p. 91.

Localité: Mudumalai, Nilgiris, 1000 m., 4.II.1927, 3 ô, 1 q. 
L'aire de distribution de cette espèce est très vaste puisqu'elle s'étend de l'Inde, par le Sud-Est de l'Asie et l'Archipel indo-australien aux îles du Pacifique occidental.

\section{Hemidactylus triedrus (Daud.).}

1890. Boulenger, G. A. Catal. of Brit. India, Rept. et Batr., p. 89. 1905. Annandale, N. L. Journ. Asiat. Soc. Bengal, vol. 1, p. 91.

Localité: Masinigudi, Nilgiris, 1000 m., 2.II.1927, 1 ○.

Cet individu possède 6 pores fémoraux de chaque côté.

Espèce habitant le centre et le Sud de l'Inde et l'île de Ceylan.

\section{Hemidactylus leschenaulti D. B.}

1890. Boulenger, G. A. Catal. of Brit. India, Rept. et Batr., p. 91. 1905. Annandale, N. L. Journ. Asiat. Soc. Bengal, vol. 1, p. 91.

Localités: Pollachi, plaine, 200 m., 21.II.1927, 1 §̊.

Coimbatore, plaine, $400 \mathrm{~m} ., 1$ s.

On compte chez ces exemplaires, 12 pores de chaque côté; leur coloration est typique.

Cette espèce est répandue dans le Sud-Est de l'Asie.

\section{Lepidodactylus aurantiacus (Bedd.).}

1890. Boulenger, G. A. Catal. of Brit. India, Rept. et Batr., p. 98. 1905. Annandale, N. L. Journ. Asiat. Soc. Bengal, vol. 1, p. 91.

Localités: Masinigudi, Nilgiris, 1000 m., 27.I.1927, 1 juv.

Attakatti, Anaimalais, 1000 m., 1.III.1927, 1 ô, 1 ㅇ.

Les taches blanches, bordées de noir qui se trouvent à la base de la queue sont très visibles.

Cette espèce est propre aux montagnes du Sud de l'Inde.

\section{Famille Agamidæ.}

\section{Sitana ponticeriana Cuv.}

1890. Boulenger, G. A. Fauna of Brit. India, Rept. et Batr., p. 114. 1905. Annandale, N. L. Journ. Asiat. Soc. Bengal, vol. 1, p. 92.

Localité: Pollachi, plaine, 200 m., 26.II.1927, 2 ๙ , 2 ․

L'aire de distribution de cette espèce comprend l'Inde et Ceylan. 


\section{Salea horsfieldi Gray.}

1890. Boulenger, G. A. Fauna of Brit. India, Rept. et Batr., p. 131. 1905. Annandale, N. Journ. Asiat. Soc. Bengal, vol. 1, p. 86, 92. 1908. Annandale, N. Records Ind. Mus., vol. 2, p. 37, 38. 1922. Wall, F. Journ. Bombay Nat. Hist. Soc., vol. 28, p. 494.

Localités: Avalanche, Nilgiris, 2100 m., 20.I.1927, 1 ㅇ, 2 juv. Ootacamund, Nilgiris, 2200 m., 12.I.1927, 2 ô, 1 juv. Bangitappali, Nilgiris, 2450 m., 26.I.1927, 4 juv.

Vandaravu, Palnis, 2350 m., IV.1927, 1 q.

Boulenger indique la région des Nilgiris comme provenance certaine et ajoute Ceylan avec un ? Annandale rapporte à cette espèce un exemplaire provenant de Moulmein, dans la Birmanie inférieure.

\section{Salea anamatlayana (Bedd.).}

1890. Boulenger, G. A. Fauna of Brit. India, Rept et Batr., p. 132. 1905. Annandale, N. Journ. Asiat. Soc. Bengal, vol. 1, p. 92. 1908. Annandale, N. Records Ind. Mus., vol. 2, p. 37, 38.

1926. Hora, S. L. Records Ind. Mus., vol. 28, p. 219.

Localités: Kodaikanal-Road, plaine, 200 m., 15.IV.1927, 1 ô.

Vandaravu, Palnis, 2350 m., 7.IV.1927, 1 ô, 2 \%, 1 juv. Pumbarai, Palnis, 2000 m., 27.III.1927, 1 ㅇ.

Ces exemplaires répondent parfaitement à la description de Boulenger, soit pour les caractères des écailles soit pour la coloration du corps.

Cette espèce qui n'était connue que du Sud de l'Inde a été signalée par Annandale à Moulmein (Birmanie inférieure).

\section{0a. Salea anamallayana triangularis $\mathbf{n}$. subsp.}

Localité: Mariyanshola, Palnis, 2300 m., 13.IV.1927, 1 ㅇ.

Cet individu se distingue au premier coup d'œil des précédents par sa coloration générale et la forme des taches dorsales. Il est d'une teinte brun rougeâtre, avec taches d'un brun tirant au noir. Tandis que chez les exemplaires ordinaires les taches dorsales sont en forme de chevrons, ici elles sont triangulaires, la base du triangle se trouvant en avant et placée transversalement à l'axe longitudinal du corps. En outre les écailles qui garnissent les flancs 
sont lisses sur 5 ou 6 rangées, au lieu d'être carénées comme les médiodorsales ou les ventrales. La crête nuchale est formée de quelques écailles lancéolées, assez distantes les unes des autres.

Nous n'avons pas trouvé chez cet individu, parmi les caractères morphologiques, des différences pouvant justifier sa séparation spécifique d'avec les exemplaires précédents.

Ce spécimen de Mariyanshola est une femelle de 21 centimètres de longueur totale, dont 8 pour la tête et le trone et 13 pour la queue.

\section{Calotes versicolor (Daud.).}

1890. Boulenger, G. A. Fauna of Brit. India, Rept. et Batr., p. 135. 1905. Annandale, N. Journ. Asiat. Soc. Bengal, vol. 1, p. 87, 92. 1926. Hora, S. L. Records Ind. Mus., vol. 28, p. 219.

Localités: Coonoor, Nilgiris, 1800 m., 20-21.XII.1926, 2 juv. Masinigudi, Nilgiris, 1000 m., II.1927, 1 ô, 1 juv. Gudalur, Nilgiris, 1100 m., II.1927, 2 ô. Mudumalai, Nilgiris, 1000 m., II.1927, 1 ô, 1 juv. Coimbatore, plaine, 400 m., III.1927, 1 oै. Pollachi, plaine, 250 m., II, 1927, 1 juv. Attakatti, Anaimalais, 1000 m., II.1927, 5 ô, 1 juv. Maryland, Palnis, 1600 m., IV.1927, 1 ô.

C'est l'espèce de Calotes qu'on rencontre le plus communément dans l'Inde; son aire de dispersion s'étend de l'Afghanistan et du Beludchistan jusqu'au Sud-Est du continent asiatique et à Sumatra.

Les jeunes ont des taches foncées transversales sur le dos, coupées par deux lignes longitudinales claires.

\section{Calotes grandisquamis Gthr.}

1890. Boulenger, G. A. Fauna of Brit India, Rept. et Batr., p. 138. 1905. Annandale, N. L. Journ. Asiat. Soc. Bengal, vol. 1, p. 93.

Localité: Valparai, Anaimalais 1100 m., 9.III.1927, 1 q.

Cet exemplaire répond bien à la description de Boulenger. Il est de couleur verte, avec 2 bandes plus foncées au travers du dos; quelques écailles de couleur jaune se trouvent de chaque côté, au-dessous du pli du cou.

Cette espèce n'est connue que du Sud-Ouest de l'Inde. 


\section{Calotes ophiomachus (Merr.).}

1890. Boulenger, G. A. Fauna of Brit. India, Rept. et Batr., p. 140. 1905. Annandale, N. L. Journ. Ásiat. Soc. Bengal, vol. 1, p. 93.

Localité: Attakatti, Anaimalais, 1000 m., II.1927, 3 ㅇ․

Ces exemplaires ont 35 à 36 écailles autour du corps; ils sont de couleur verte, avec 4 ou 5 bandes étroites, de couleur claire (jaune dans l'alcool) au travers du dos.

L'aire de dispersion de cette espèce comprend le Sud de l'Inde, Ceylan et les Nicobars, d'après Boulenger.

\section{Calotes ellioti Gthr.}

1890. Boulenger, G. A. Fauna of Brit. India, Rept. et Batr., p. 142. 1905. Annandale, N. L. Journ. Asiat. Soc. Bengal, vol. 1, p. 93.

Localités: Coonoor, Nilgiris, 1800 m., 9.I.1927, 1 ô. Valparai, Anaimalais, 1100 m., 9.III.1927, 1 ô, 1 ㅇ. Tandikudi, Palnis, 1500 m. IV.1927, 1 $\widehat{\jmath}$.

Ces spécimens concordent bien avec la description donnée par Boulenger. Chez le ô de Valparai, la couleur générale du corps est plus foncée sur le dos et les branches transversales sont partant moins visibles. Les côtés de la tête et du cou sont très foncés et la tache suboculaire claire très apparente, ainsi que 2 ou 3 écailles isolées, claires également, sur les côtés de la gorge. Le menton et la partie médiane de la gorge sont d'un gris clair.

Cette espèce est propre au Sud de l'Inde. On voit, par les localités citées, qu'elle existe dans les trois groupes montagneux.

\section{Charasia dorsalis (Gray).}

1890. Boulenger, G. A. Fauna of Brit. India, Rept. et Batr., p. 142. 1905. Annandale, N. L. Journ. Asiat. Soc. Bengal, vol. 1, p. 93. 1912. Annandale, N. L. Records Ind. Museum, vol. 7, p. 46. 1922. Wall, F. Journ. Bombay Nat. Hist. Soc., vol. 28, p. 493.

Localités: Coonoor, Nilgiris, 1800 m., XII.1926, 2 ô, 1 ๆ, 2 juv. Attakatti, Anaimalais, 1000 m., II.1927, 4 ô, 1 ㅇ Tandikudi, Palnis, 1500 m., IV.1927, 1 q. Maryland, Palnis, 1600 m., 19.IV.1927, 1 ô. 
Les exemplaires jeunes et les femelles sont bruns, avec taches claires disséminées sur le dos. Queue d'un brun noirâtre, avec anneaux plus clairs, plus étroits que les espaces qui les séparent. La tête est brune, mêlée de gris. Les mâles sont d'un gris jaunâtre, avec une large bande latérale foncée, commençant en arrière de l'œil.

Cette espèce n'est connue que du Sud de l'Inde, dans les montagnes.

\section{Famille Lacertidæ.}

36. Cabrita leschenaulti (M. Edw.).

1890. Boulenger, G. A. Fauna of Brit. India, Rept. et Batr., p. 172. 1905. Annandale, N. L. Journ. Asiat. Soc. Bengal, vol. 1, p. 149.

Localités: Pollachi, plaine, 250 m., 26.II.1927, 1 Ex. Attakatti, Anaimalais, 1000 m., II.1927, 1 Ex.

Cette espèce habite la partie péninsulaire de l'Inde et Ceylan.

\section{Famille Scincidæ.}

\section{Mabuia bibroni (Gray).}

1890. Boulenger, G. A. Fauna of Brit. India, Rept. et Batr., p. 184. 1905. Annandale, N. L. Journ. Asiat. Soc. Bengal, vol. 1, p. 149. 1912. Annandale, N. L. Records Ind. Mus., vol. 7, p. 90, 91. 1927. Hora, S. L. Records Ind. Mus., vol. 29, p. 1, 2.

Localité: Coimbatore, plaine, 400 m., 2 ㅇ.

Ces exemplaires, dont la coloration est typique, possèdent 30 écailles autour du corps; les écailles dorsales sont munies de 7 fortes carènes.

Cette espèce est propre au Sud-Est de la péninsule indoue et à Ceylan. Annand ale pensait que cette espèce était liée au voisinage des côtes, mais depuis lors, il l'a trouvée précisément dans la même localité d'où provient notre exemplaire, ainsi que le rapporte Hora. Elle est donc répandue aussi dans les régions inférieures de l'intérieur de la péninsule. Le fait qu'elle n'a pas été rencontrée sur la côte de Travancore s'explique, d'après HorA, par le fait qu'elle n'a pas pu traverser les chaînes de montagnes du Sud de la péninsule.

Rev. Suisse de Zool. T. 35, 1928. 


\section{Mabuia carinata (Schn.).}

1890. Boulenger, G. A. Fauna of Brit. India, Rept. et Batr., p. 188.

Localités: Pollachi, plaine, 21.II.1927, 1 juv.

Valparai, Anaimalais, 1100 m., 9.III.1927, 2 ô.

Ces individus, dont le plus grand mesure $125^{\mathrm{mm}}$ du museau à l'anus, ont 32 écailles autour du corps.

L'espèce, très commune, au dire de Boulenger, est répandue dans l'Inde, à Ceylan et en Birmanie.

\section{Mabuia macularia (Blyth).}

1890. Boulenger, G. A. Fauna of Brit. India, Rept. et Batr., p. 189. 1927. Hora, S. L. Records Ind. Museum, vol. 29, p. 2.

Localités: Masinigudi, Nilgiris, 1000 m., 28.I.1927, 2 శ.

Mudumalai, Nilgiris, 1000 m., 3.II.1927, 1 Ex.

Attakatti, Anaimalais, 1000 m., 24.II.1927, 1 Ex.

Valparai, Anaimalais, 1100 m., 9.III.1927, 1 Ex.

Ces spécimens ont 30 écailles autour du corps, ils sont d'un brun uniforme en dessus; les côtés du corps sont un peu plus clairs et peuvent porter quelques taches blanches sur quelques écailles. La face inférieure est blanche, avec des lignes longitudinales grises, peu accusées, marquant les côtés des écailles.

Hor a dit de cette espèce qu'elle est assurément la plus commune de l'Inde. Sa distribution s'étend jusqu'à la base de l'Himalaya occidental; on la trouve aussi à Ceylan, et dans le Sud-Est du continent asiatique.

\section{Lygosoma (Liolepisma) bilineatum (Gray).}

1890. Boulenger, G. A. Fauna of Brit. India, Rept. et Batr., p. 202. 1905. Annandale, N. L. Journ. Asiat. Soc. Bengal, vol. 1, 1905, p. 150.

Localités: Avalanche, Nilgiris, 2100 m., 18.I.1927, 2 Ex.

Bangitappali, Nilgiris, 2450 m., 20.I.1927, 6 Ex.

Ces exemplaires ont 24-26 écailles autour du corps; le plus grand mesure $145 \mathrm{~mm}$ de longueur totale, dont $52^{\mathrm{mm}}$ pour la tête et le corps et 93 pour la queue.

Cette espèce semble être spéciale au massif des Nilgiris. 


\section{Lygosoma (Liolepisma) travancoricum (Bedd.).}

1890. Boulenger, G. A. Fauna of Brit. India, Rept. et Batr., p. 204. 1927. Hora, S. L. Journ. Asiat. Soc. Bengal, vol. 29, p. 5.

Localités: Kodaikanal, Palnis, 2200 m., 25.III.1927, 2 Ex. Pumbarai, Palnis, 2000 m., 30.III.1927, 3 Ex. Vandaravu, Palnis, 2350 m., 5.IV.1927, 1 Ex. Mariyanshola, Palnis, 2500 m., 17.IV. 1927,2 Ex. Maryland, Palnis, 1600 m., 20.IV.1927, 2 Ex. Tandikudi, Palnis, 1500 m., 23.IV.127, 4 Ex.

Cette espèce, propre au Sud de l'Inde semble n'habiter que les régions montagneuses.

\section{Lygosoma (Riopa)punctatum (L.).}

1890. Boulenger, G. A. Fauna of Brit. India, Rept. et Batr., p. 208. 1905. Annandale, N. L. Journ. Asiat. Soc. Bengal, vol. 1, p. 150.

Localités: Coimbatore, plaine, 400 m., 16.XII.1926, 3 Ex. Coonoor, Nilgiris, 1800 m., 20.XII.1926, 2 Ex. Attakatti, Anaimalais, 1000 m., 22.II.1927, 1 Ex.

Le plus grand des exemplaires de Coimbatore mesure $140 \mathrm{~mm}$ de longueur totale. L'écaillure de la tête est normale, les deux plaques supranasales forment une courte suture en arrière de la rostrale, séparant cette dernière de la frontonasale. En arrière des pariétales une paire de plaques nuchales. On compte 26 écailles autour du corps. La couleur générale est d'un brun clair, les écailles dorsales sont munies en leur partie centrale d'une tache noire. La bande dorsolatérale claire est assez bien visible.

Les deux autres spécimens ont également 26 écailles autour du tronc. Chez l'un d'eux, l'une des plaques nuchales est divisée en 2. Les taches noires, des écailles forment 6 bandes longitudinales et la raie claire dorso-latérale est bien accusée.

L'un des spécimens de Coonoor est un jeune qui possède également 26 écailles autour du corps. La disposition des plaques de la tête est normale, de même que la coloration. Chez cet individu, les taches noires des écailles dorsales sont réunies et forment 6 bandes longitudinales. La raie claire dorso-latérale est très visible; elle 
commence sur le museau et se poursuit en passant au-dessus de l'œèil jusqu'à la base de la queue. Cette dernière a la couleur rosée, caractéristique pour le jeune de cette espèce.

L'autre individu de Coonoor présente 4 plaques nuchales au lieu de 2 ; il possède 28 écailles autour du corps et sa teinte générale est plus foncée que chez les précédents, elle est plutôt d'un brungrisâtre. La raie dorso-latérale claire est peu visible. La queue est de même couleur que le dos.

Cette même coloration se retrouve chez l'exemplaire d'Attakatti qui ne possède, lui, que 24 écailles autour du corps. Chez cet individu les supra-nasales sont légèrement séparées l'une de l'autre au lieu de former une suture. Les nuchales sont au nombre normal de 2 .

Chez tous ces exemplaires la face ventrale est blanche.

Cette espèce est commune dans la partie péninsulaire de l'Inde et se trouve également à Ceylan.

\section{Ristella rurki Gray.}

1890. Boulenger, G. A. Fauna of Brit. India, Rept. et Batr., p. 215. 1905. Annandale, N. L. Journ. Asiat. Soc. Bengal, vol. 1, p. 150. 1927. Hora, S. L. Records Ind. Museum, vol. 29, p. 6.

Localités: Kukkal, Palnis, 1900 m., IV.1927, 2 Ex. Pumbarai, Palnis, 2000 m., 28.III.1927, 2 Ex.

Chez ces exemplaires, dont la coloration est normale, les écailles dorsales sont lisses.

Cette espèce est propre aux régions montagneuses du Sud de l'Inde, comme du reste le genre Ristella qui se distingue du genre précédent par des ongles rétractiles dans une gaine terminant les doigts et les orteils.

\section{BATRACHIA}

\section{Famille Ranidæ.}

\section{Rana cyanophlyctis Schn.}

1890. Boulenger, G. A. Fauna of Brit. India, Rept. et Batr., p. 442. 1920. Boulenger, G. A. Records Ind. Museum, vol. 20, p. 12 (bibliographie).

1923. Hora S. L. et Chopra, B. Records Ind. Museum, vol. 25, p. 376. 
Localité: 'Tandikudi, Palnis, 1500 m., 23.IV.1927, 1 ㅇ.

Cet exemplaire mesure $45 \mathrm{~mm}$ du bout du museau à l'anus. Les larges membranes séparant les orteils sont tachées de gris foncé. Cette espèce, qui est franchement aquatique et ne craint pas les eaux saumâtres, est très commune dans les plaines de l'Inde mais se rencontre aussi dans les divers massifs montagneux. Son aire de dispersion s'étend de l'Arabie méridionale à la presqu'ìle malaise.

\section{Rana verrucosa (Gthr).}

1890. Boulenger, G. A. Fauna of Brit. India, Rept. et Batr., p. 448. 1920. Boulenger, G. A. Records Ind. Museum, vol. 20, p. 26 (bibliographie).

Localités: Valparai, Anaimalais, 1100 m., 4.III.1927, 1 ㅇ.

Tandikudi, Palnis, 1500 m., 23.IV.1927, 1 ․

Cette espèce est propre au Sud de l'Inde; elle n'a été signalée jusqu'ici que des collines de la côte de Malabar et des Nilgiris; comme on le voit elle existe aussi dans les massifs situés plus avant dans l'intérieur de la région méridionale de la péninsule.

\section{Rana limnocharis nilagirica Jerd.}

1853. R. nilagirica, Jerdon, J. C. Journ. Asiat. Soc. Bengal, vol. 22, p. 532.

1905. R. nilagirica. Boulenger, G. A. Spolia zeylon, vol. 2, p. 73. 1916. R. limn. nilagirica. Annandale, S. L. Mem. Asiat. Soc. Bengal, vol. 6, p. 134 (bibliographie).

1920. R. limn. nilagirica. Boulenger, G. A. Records. Ind. Mus., vol. 20 , p. 33 .

Localités: Coonoor, Niggiris, 1800 m., 18.XII.1926, 1 ㅇ.

Gudalur, Nilgiris, 1100 m., 11-14.II.1927, 1 q, 1 juv.

Masinigudi, Nilgiris, 1000 m., 27.I-1.II.1927, 3 juv.

Mudumalai, Nilgiris, 1000 m., 7.II.1927, 2 juv.

Attakatti, Anaimalais, 1000 m., 27.II.1927, 2 juv.

Vandaravu, Palnis, 2350 m., 8.IV.1927, 1 juv.

Je rapporte à cette sous-espèce de $R$. limnocharis tous ces individus dont le membre postérieur est un peu plus long que chez 
la forme type. L'articulation tibio-tarsienne atteint le bout du museau chez les jeunes, tandis qu'elle atteint au delà de ce point chez les 2 ㅇ. Les orteils sont à demi-palmés. Un tubercule métatarsien externe est distinct chez la plupart des exemplaires, ainsi que le pli tarsien qui est court.

La description initiale de JERDON est malheureusement très courte; il me paraît que Boulenger et ANNANDALF ont raison de considérer comme une sous-espèce de Rana limnocharis la forme qui a été distinguée par JERDon, sous le nom de nilagirica.

Nous rapporions également à cette sous-es ${ }_{i}^{-}$:e deux individus jeunes provenant des Anaimalais qui présentent les mêmes caractères que ceux des Nilgiris, mais dont la livrée générale est plus sombre.

Un jeune individu des Palnis est en tout semblable aux jeunes des Nilgiris et nous semble appartenir aussi à cette sous-espèce, qui serait ainsi distribuée dans les régions montagneuses du Sud de l'Inde.

\section{6a. Rana limnocharis parvipalmata Ptrs.}

1871. R. parvipalmata. Peters, W. Monatsber. Berlin Acad., p. 646. 1920. R. parvipalmata. Boulenger, G. A. Records Ind. Mus., vol. 20, p. 37 (bibliographie).

Localité: Gudalur, Nilgiris, 1100 m., 12.II.1927, 1 ㅇ․

Parmi les Rana limnocharis des Nilgiris que nous avons examinées, se trouve un spécimen qui répond parfaitement à la description initiale de P.eters de $R$. parvipalmata. Cette forme ne se distingue de $R$. limnocharis que par la membrane moins développée aux orteils; ceux-ci ne sont en effet palmés que dans leur tiers proximal. Les autres caractères étant en tout ceux de $R$. limnocharis nılagirica; il me semble plus juste de ne considérer l'espèce de Peters que comme sous-espèce de Rana limnocharis. Chez cet individu le museau est un peu plus pointu que chez les autres et le tubercule métatarsien externe est absent de même que le pli du tarse.

Cette sous-espèce habite la même région que la précédente.

\section{Rana beddomei (Gthr).}

1890. Boulenger, G. A. Fauna of Brit. India, Rept. et Batr., p. 453. 1920. Boulenger, G. A. Records Ind. Museum, vol. 29, p. 114 (bibliographie). 
Localités: Attakatti, Anaimalais, 1100 m., 23.II.1927, 6 juv. Valparai, Anaimalais, 1100 m., 9-11.III.1927, 3 juv. Tandikudi, Palnis, 1500 m., 23.IV.1927, 2 \%, 1 juv.

Ces exemplaires répondent bien à la description de Boulenger.

La coloration est de teinte générale gris légèrement rosé chez les 2 o de Tandikudi, tandis que les jeunes sont d'un gris plus franc. Parmi les jeunes d'Attakatti, 3 possèdent une large bande vertébrale blanche. Les taches noires bordant le canthus rostralis et celles de la région tympanique sont toujours très apparentes ainsi que celles deg bords de la bouche. La barre transversale interorbitaire, les taches dorsales et les bandes transversales ornant les membres sont de teinte brune et plus ou moins apparentes.

Cette espèce est particulière aux régions montagneuses du Sud de l'Inde.

\section{Rana temporalis Gthr.}

1890. Boulenger, G. A. Fauna of Brit. India, Rept. et Batr., p. 459 1920. Boulenger, G. A. Records Ind. Museum, vol. 20, p. 159 (bibliographie).

1922. Wall, F. Jour. Bombay Nat. Hist. Soc., vol. 28, p. 498.

Localités: Masinigudi, Nilgiris, 1000 m., 31.I.1927, 1 juv. Mudumalai, Nilgiris, 1000 m., 7-II.1927, 1 ô juv.

Attakatti, Anaimalais, 1000 m., 24.II.1927, 1 o.

Valparai, Anaimalais, 1100 m., 11.III.1927, 5 ô.

Tandikudi, Palnis, 1500 m., 23.IV.1927, 1 ô.

La plupart de ces exemplaires sont encore jeunes; la plus grande + de Valparai atteint $65 \mathrm{~mm}$ (distance du museau à l'anus). Le dessus du corps est brun uniforme, les faces latérales d'un brun noir; la ligne blanche bordant la lèvre supérieure est toujours très visible.

Cette espèce habite le Sud de l'Inde et Ceylan.

\section{Micrixalus silvaticus Blgr.}

1890. Boulenger, G. A. Fauna of Brit. India, Rept. et Batr., p. 466.

Localités: Mudumalai, Nilgiris, 1000 m., 7.II.1927, 1 Ex.

Kukkal, Palnis, 1900 m., 1.IV.1927, 1 Ex.

Mariyanshola, Palnis, 2300 m., 14.IV.1927, 1 Ex. 
Chez l'exemplaire de Kukkal, la partie antérieure des cuisses et la région de l'aine ont gardé des restes de la coloration rose de l'animal vivant.

Cette espèce habite les forêts montagneuses du Sud de l'Inde. Elle était connue des collines de la côte de Malabar; on voit qu'elle se rencontre dans le massif des Palnis, à d'assez grandes altitudes.

\section{Nyctibatrachus major Blgr.}

1890. Boulenger, G. A. Fauna of Brit. India, Rept. et Batr., p. 468. 1904. Ferguson, H. S. Journ. Bombay Nat. Hist. Soc., vol. 15, p. 503.

Localité: Masinigudi, Nilgiris, 1000 m., 31.I.1927, 1 ㅇ.

Chez cet individu, l'articulation tibio-tarsienne atteint le bord postérieur de l'œil. Les œufs sont gros, ils mesurent chez cette $q$ de $36 \mathrm{~mm}$ (museau-anus) $2^{\mathrm{mm}}$ de diamètre.

Chez le genre Nyctibatrachus l'omosternum est fourchu à sa base. Cette espèce est limitée au Sud de la péninsule indoue.

\section{Rhacophorus maculatus (Gr.).}

1890. Boulenger, G. A. Fauna of Brit. India, Rept. et Batr., p. 475, 1904. Ferguson, H. S. Journ. Bombay Nat. Hist. Soc., vol. 15, p. 504. 1918. Annandale, N. L. et Rao, N. C. R. Records Ind. Mus., vol. 15, p. 36 (larve).

Localités: Ootacamund, Nilgiris, 2200 m., 12.I.1927, 2 ㅇ. Pumbarai, Palnis, 2000 m., 29.III.1927, 1 juv.

Cette espèce est très répandue, en montagne comme dans les régions basses; son aire de dispersion comprend l'Inde et Ceylan.

\section{Rhacophorus pleurostictus (Gthr).}

1890. Boulenger, G. A. Fauna of Brit. India, Rept. et Batr., p. 479.

Locaités: Conoor, Nilgiris, 1800 m., 19.XII.1926, 1 juv.; Bangitappali, Nilgiris,2500 m., 19.I.1927, 2 juv.

Cette espèce est connue de la région des monts Nilgiris et Anaimalais. 


\section{Philautus leucorhinus (Mart.)}

1890. Ixalus leucorhinus. Boulenger, G. A. Fauna of Brit. India, Rept. et Batr., p. 483.

1904. Ixalus leucorhinus. Ferguson, H. S. Journ. Bombay Nat. Hist. Soc., vol. 15, p. 505.

Localités: Mudumalai, Nilgiris, 1000 m., 7.II.1927, 1 juv.

Valparai, Anaimalais, 1100 m., 3.III.1927, 3 Ex. juv.

Ces exemplaires concordent assez bien avec ceux de Ceylan que possède le Musée de Bâle, aussi les rapportons-nous à cette espèce malgré quelques différences avec la description que donne Boulenger (il ne nous a pas été possible de consulter la diagnose initiale de von Martens). Le tympan est visible, mais il mesure à peine la moitié de l'orbite, parfois moins encore. Les doigts sont libres; quant aux orteils, ils ne sont pas tout à fait à demi-palmés, la membrane est plus étendue entre les orteils externes qu'entre les internes. Elle est très échancrée et occupe un peu plus du tiers de la longueur entre les orteils 3 et 4 et 4 et 5 , par contre elle est un peu moins développée entre les orteils 2 et 3 et encore moins entre les orteils 1 et 2. Les disques terminaux sont bien développés. L'articulation tibio-tarsienne atteint entre l'œil et le museau. La peau est lisse en dessus, de même sous la gorge, mais elle est fortement granuleuse sur le ventre et la face inférieure des cuisses. Un léger pli se remarque au-dessus du tympan.

La coloration semble varier passablement chez cette espèce, tout comme chez $P$. variabilis, si j'en juge par les spécimens de Ceylan que j'ai sous les yeux et ces spécimens du Sud de l'Inde. A côté d'individus bruns, plus ou moins foncés, il s'en trouve des gris, d'autres presque blancs.

Le spécimen de Mudumalai est d'un gris très clair; de petites taches et points noirs sont irrégulièrement disséminés sur le dos. Une large bande interoculaire brunâtre se perçoit indistinctement ainsi que les bandes transversales sur les membres. La région située sous le canthus rostralis est brunâtre et la tache la mieux marquée est celle qui, de couleur noire, couvre en partie le tympan et se prolonge un peu en arrière, en s'amincissant, du côté de l'épaule.

Chez les individus de Valparai, la couleur générale est d'un gris plus foncé que chez l'exemplaire précédemment décrit. Les 
taches de la face dorsale sont plus accusées; on aperçoit une bande foncée entre les orbites et deux bandes légèrement arquées, allongées, une de chaque côté de la région médiane. Les lèvres sont tachées de brun et la gorge piquée de la même teinte. Le dessus des cuisses et du tibia est orné de quelques bandes transversales foncées; la partie postérieure des cuisses est brunâtre. Le ventre est blanc uniforme ou maculé de brun.

Cette espèce est connue des montagnes du Sud de l'Inde et de Ceylan.

\section{Philautus variabilis (Gthr.)}

1890. Ixalus variabilis. Boulenger, G. A. Fauna of Brit. India, Rept. et Batr., p. 487.

1904. Ixalus variabilis. Ferguson, H. S. Journ. Bombay Nat. Hist. Soc., vol. 15, p. 505.

1922. Ixalus variabilis. Wall, F. Journ. Bombay Nat. Hist. Soc., vol. 28, p. 494.

Localités: Maryland, Palnis, 20.IV.1927, 1600 m., 1 juv.

Kukkal, Palnis, 1-2.IV.1927, 1900 m., 3 juv.

Mariyanshola, Palnis, 11.IV.1927, 2500 m., 1 juv.

Chez ces spécimens, dont le plus grand mesure $26^{\mathrm{mm}}$, on peut reconnaitre la plupart des caractères de l'espèce de Günther. La langue ne possède pas de grande papille libre dans la portion antérieure, le tympan est médiocrement visible dans sa partie inférieure. La peau est pourvue de petits tubercules irrégulièrement distribués sur le dos et les côtés; la face inférieure du corps, y compris la gorge est granuleuse. En comparant ces individus avec ceux de Ceylan, j'ai pu me rendre compte qu'il peut y avoir une certaine variation dans le développement de la membrane entre les orteils. C'est à peine si celle-ci s'étend jusqu'à la moitié des orteils externes (3-4 et 4-5) et elle ne réunit que la base des orteils internes (1-2 et 2-3). Les doigts sont libres et les disques, assez bien développés, sont un peu plus grands aux doigts qu'aux orteils.

L'articulation tibio-tarsienne atteint l'œil.

Les auteurs ont relevé la grande diversité de coloration que présente cette espèce, caractère qui lui a valu sa dénomination spécifique. Nos exemplaires des Palnis en sont une nouvelle démonstration. Chez les spécimens de Maryland et le Mariyanshola le corps est d'un gris plutôt clair, uniforme sur le dos. Dans la région 
de l'aine quelques marbrures brunâtres qui s'étendent aussi sur le côté de la cuisse. Quelques taches peu accusées oceupent la face supérieure du pied. Les côtés de la tête sont plus foncés que le dessus du museau. La face inférieure du corps est blanchâtre.

Un des exemplaires de Kukkal est d'un gris un peu plus foncé, avec taches noirâtres sur le dos; les côtés de la tête sont bordés de noir le long du canthus rostralis et dans la région du tympan; les taches de la région de l'aine sont bien visibles et les membres présentent quelques larges bandes transversales ainsi que des macules irréguliers de couleur noirâtre. Sur la gorge, de larges marbrures foncées; ventre d'un gris blanchâtre.

Les deux autres individus de Kukkal sont d'un brun foncé, avec des taches bien marquées, soit une bande interoculaire et des dessins méandriques sur le dos qui rappellent ceux de Ph. (Ixalus) brevipes Blgr. ${ }^{1}$. Les taches et marbrures des côtés de la tête, de la région inguinale et de la partie postérieure des cuisses sont bien accusées, de même que les bandes ornant les membres. La gorge est plus ou moins lavée de brun, tandis que le ventre est grisâtre.

Cette espèce est connue du Sud de l'Inde et de Ceylan.

\section{Philautus glandulosus (Jerd.).}

1890. Ixalus glandulosus. Boulenger, G. A. Fauna of Brit. India, Rept. et Batr., p. 488.

1904. Ixalus glandulosus. Ferguson, H. S. Journ. Bombay Nat. Hist. Soc., vol. 15, p. 505.

1922. Ixalus glandulosus. WALL, F. Journ. Bombay Nat. Hist. Soc., vol. 28, p. 497.

Localités : Coonoor, Nilgiris, 1800 m., 24.XII.1926, 4 Ex.

Avalanche, Nilgiris, 2100 m., 12.I.1927, 3 Ex.

Ootacamund, Nilgiris, 2200 m., 12-14.I.1927, 11 Ex.

Chez cette espèce, la langue est pourvue dans sa partie antérieure d'une papille libre, bien visible. Le museau est arrondi, le tympan caché; les doigts sont libres, les orteils externes palmés sur leur tiers proximal; les autres à la base seulement. Les disques terminaux sont bien développés. L'articulation tibio-tarsienne atteint l'œil.

${ }^{1}$ Boulenger G.A. in: Journ. Feder. Malay. States Museum, Vol. 3, 1908, p. 63. Pl. IV. Fig. 1. 
La peau est en général lisse sur le dos, mais les côtés du corps présentent de nombreux petits tubercules glanduleux. Ces exemplaires présentent, comme l'espèce précédente, de grandes différences de coloration. Les uns sont d'un gris uniforme sur le dos, avec aréolation un peu plus claire sur les côtés du corps. La face latérale de la tête et la région tympanique sont de teinte plus foncée. La région inguinale est brune ou noire, avec ou sans taches claires, de même la partie postérieure des cuisses.

Chez d'autres, le dos porte des taches foncées dont les plus constantes sont une barre interoculaire et 2 taches allongées, plus ou moins symétriques, arquées en dedans, sur le dos. Les membres sont ornés de nombreuses bandes transversales foncées, étroites qu'on peut apercevoir jusque sur le dessus des pieds. La face ventrale, granuleuse, est d'un gris plus ou moins taché ou aréolé de brun.

Cette espèce est spéciale aux districts montagneux du Sud de l'Inde et semble n'habiter que les régions élevées.

\section{Famille Brevicipitidæ (Engystomatidæ)}

\section{Melanobatrachus indicus Bedd.}

1890. Boulenger, G. A. Fauna of Brit. India, Rept. et Batr., p. 489. 1904. Ferguson, H. S. Journ. Bombay Nat. Hist. Soc., vol. 15, p. 505.

Localité: Valparai, Anaimalais, 1100 m., 4.III.1927, 1 Ex.

Cet individu, absolument typique, a une longueur de $28 \mathrm{~mm}$ (museau-anus). L'espèce et le genre lui-même sont particuliers à la région méridionale de l'Inde où ils semblent confinés dans la partie occidentale.

57. Microhyla rubra (Jerd.).

1890. Boulenger, G. A. Fauna of Brit. India, Rept. et Batr., p. 491. 1904. Ferguson, H. S. Journ. Bombay Nat. Hist. Soc., vol. 15, p. 506. 1918. Rao, C. R. N. Records Ind. Mus., vol. 15, p. 42 (larve).

Localité: Pollachi, 250 m., 26.II.1927, 2 Ex.

Cette espèce habite les régions basses et est répandue dans l'Inde et à Ceylan. 


\section{Famille Bufonidæ.}

58. Bufo melanostictus Schn.

1890. Boulenger, G. A. Fauna of Brit. India, Rept. et Batr, p. 505, fig. 140.

1904. Ferguson, H. S. Journ. Bombay Nat. Hist. Soc., vol. 15, p. 508. 1918. Annandale, N. et RaO, C. R. N. Records Ind. Mus., vol. 15, p. 38 (larve).

1922. Wall, F. Journ. Bombay Nat. Hist. Soc., vol. 28, p. 499.

Localités: Masinigudi, Nilgiris, 1000 m., 27.I.1927, 7 juv. Gudalur, Nilgiris, 1100 m., 10.II.1927, 1 §ै, 2 juv.

Coonoor, Nilgiris, 1800 m., 11-25.XII.1926, 7 ^^, 1 ㅇ. Valparai, Anaimalais, 1100 m., 5.III.1927, 1 juv.

Cette espèce est l'un des Amphibiens les plus communs de l'Inde, de Ceylan et de toute la partie sud-orientale de l'Asie, y compris l'Archipel malais. Elle habite la plaine comme aussi les régions montagneuses.

\section{Famille Cœciliidæ.}

59. Uraeotyphlus oxyurus (Dum. et Bibr.).

1890. Boulenger, G. A. Fauna of Brit. India, Rept. et Batr., p. 517. 1904. Ferguson, H. S. Journ. Bombay Nat. Hist. Soc, vol. 15, p. 509. 1913. Nieden, Fr. Gymnophiona in: Tierreich, Berlin, p. 16.

Localité: Maryland, Palnis, 1600 m., IV.1927, 5 Ex.

Le plus grand de ces exemplaires a une longueur totale de 265 millimètres. Il est d'un gris brunâtre en dessus et d'un gris de plomb sur la face ventrale. Les plis circulaires sont au nombre de 204; ils sont alternativement plus longs et plus courts, mais laissent tous libre la région médio-ventrale.

Cette espèce, est particulière aux régions montagneuses du Sud de l'Inde. 
Liste des espèces récoltées, réparties selon les provenances ${ }^{1}$.

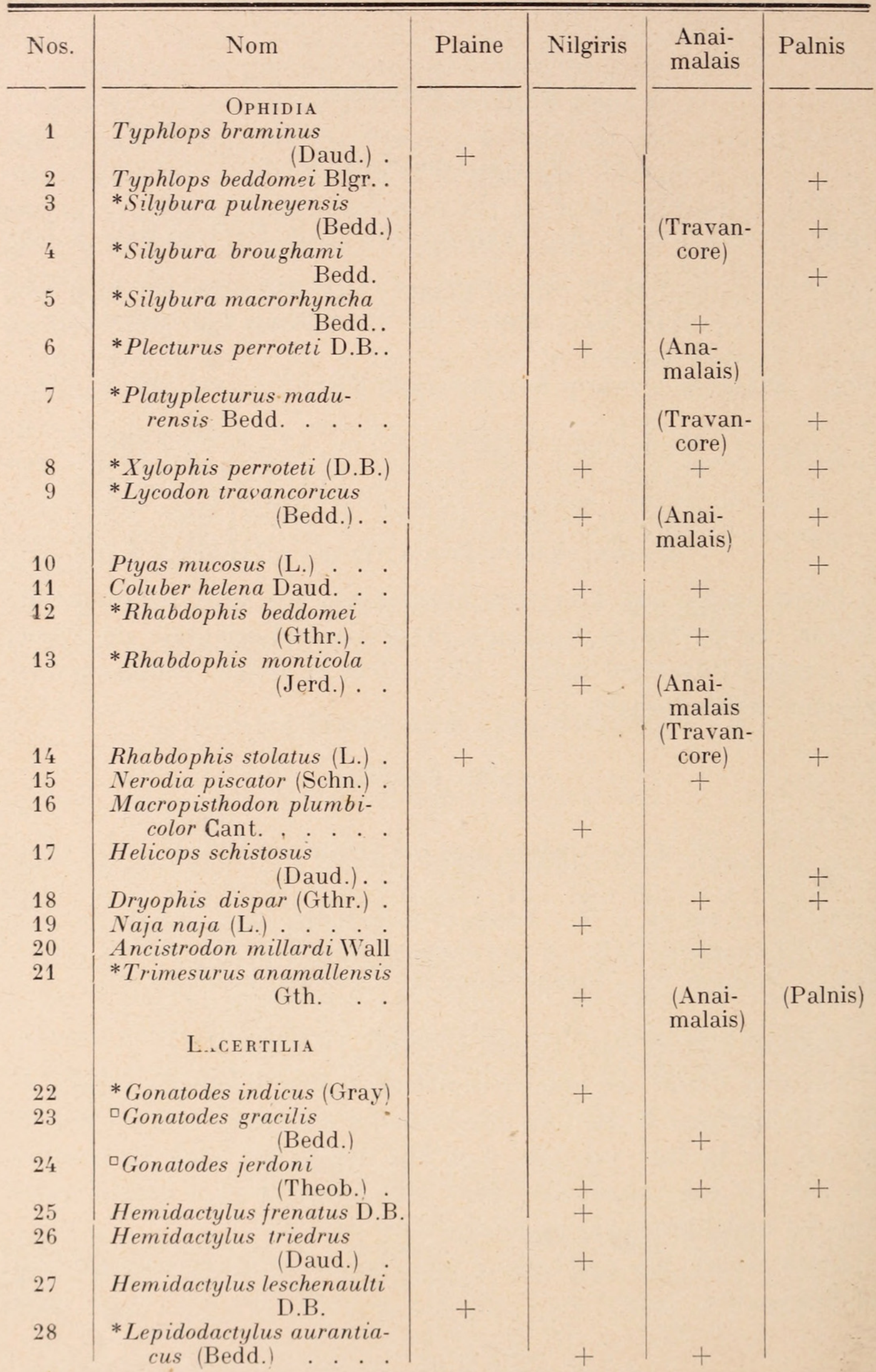

* Espèces strictement endémiques (Inde méridionale).

- " endémiques régionales (Inde méridionale et Ceylan).

1 Les provenances placées entre parenthèses dans les colonnes sont tirées de la bibliographie. 


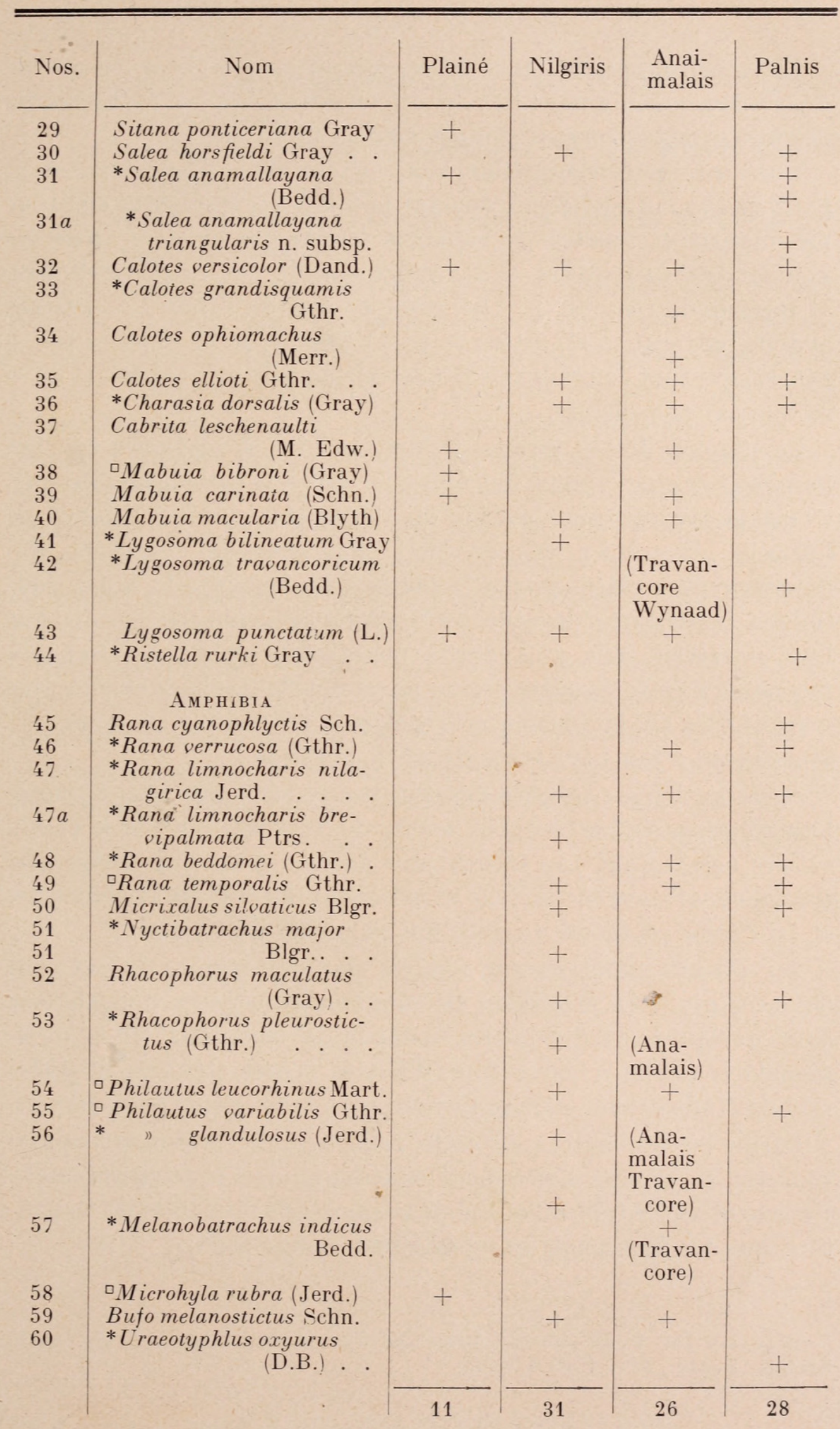


Cette collection renferme 60 espèces et sous-espèces, ce qui ne représente, comme nous l'avons dit au début, qu'une faible partie des formes actuellement connues du Sud de l'Inde.

Dans son intéressante étude sur l'histoire de la faune de Ceylan, Fr. Sarasin ${ }^{1}$ a également analysé la faune erpétologique de l'Inde méridionale et montré les rapports étroits qui existent entre les populations de Reptiles et d'Amphibiens de ces deux territoires. Il mentionne (loc. cit. p. 136) pour le Sud de l'Inde 136 espèces endémiques qui se répartissent en 11 genres et 17 espèces (en 3 genres) qui sont communes à l'Inde méridionale et à Ceylan et ne se retrouvent pas ailleurs.

La plupart de ces formes appartiennent à d'anciennes couches faunistiques que l'auteur fait remonter soit à la période mésozoïque, avant la transgression marine du Cénomanien, soit au début de l'Eocène. Par suite des énormes épanchements trappéens qui recouvrirent à cette époque le plateau du Dekkan, la faune sudindoue fut séparée de celle du Nord de la péninsule, tandis que Ceylan restait relié à la partie méridionale de l'Inde. Sarasin reconnaît à cette dernière région un caractère insulaire encore plus marqué qu'à Ceylan, bien que le Sud de la péninsule n'ait jamais été séparé tout à fait par la mer du reste de l'Inde.

A ces anciennes couches faunistiques se sont associées de nombreuses espèces plus récentes, venues du Nord, surtout par la région côtière occidentale. Les formes plus anciennes se sont conservées, spécialement dans les massifs montagneux du Sud de la péninsule, et lui confèrent encore aujourd'hui un cachet particulier.

Ces massifs montagneux, séparés par des régions basses, ont été propices à la formation de nombreuses espèces particulières dans quelques-uns des genres endémiques régionaux (Inde méridionale et Ceylan); par contre d'autres genres strictement endémiques (Inde méridionale ou Ceylan) sont restés pauvres en espèces.

Parmi les 60 espèces de cette collection, 29 seulement appartiennent au groupe strictement endémique de l'Inde méridionale. En consultant le tableau que nous avons donné plus haut on constatera que ces 29 espèces se répartissent comme suit:

${ }^{1}$ Sarasin Fr. Ueber die Geschichte der Tierwelt von Ceylon. Zool. Jahrb. System. Suppl. XII. Heft 1. 1910. 
1) 5 espèces ont été trouvées dans les trois massifs montagneux parcourus.

2) 6 espèces ont été trouvées dans deux des massifs montagneux parcourus.

3) 18 espèces ont été trouvées dans un seul des massifs montagneux parcourus.

On pourrait conclure de ces chiffres une localisation bien accusée pour la majorité des espèces endémiques récoltées. Cependant, en consultant les travaux erpétologiques qui étaient à notre disposition, nous avons constaté que plusieurs des espèces que nous avons dû classer sous le chiffre 3, appartiennent en réalité au groupe 2, d'autres passant du groupe 2 au groupe 1 ; ces indications complémentaires sont placées entre parenthèses dans le tableau de répartition et ne sont données que pour les espèces endémiques.

En résumé, cette collection renferme quelques espèces réparties dans tout le Sud-Est de l'Asie, d'autres espèces dont la distribution se limite au Sud de l'Inde et à Ceylan et des formes strictement endémiques, accusant une localisation plus ou moins marquée dans les massifs montagneux. Etant donné l'état fragmentaire des récoltes, il est impossible de préciser davantage les différences que peuvent présenter entre eux les massifs montagneux étudiés, pour ce qui a trait à leur population erpétologique. 


\section{$2 \mathrm{BHL}$ Biodiversity Heritage Library}

1928. "Reptiles et Amphibiens de l'Inde meridionale." Revue suisse de zoologie 35, 439-471. https://doi.org/10.5962/bhl.part.117624.

View This Item Online: https://www.biodiversitylibrary.org/item/148556

DOI: https://doi.org/10.5962/bhl.part.117624

Permalink: https://www.biodiversitylibrary.org/partpdf/117624

\section{Holding Institution}

American Museum of Natural History Library

\section{Sponsored by}

BHL-SIL-FEDLINK

\section{Copyright \& Reuse}

Copyright Status: In copyright. Digitized with the permission of the rights holder.

Rights Holder: Muséum d'histoire naturelle - Ville de Genève

This document was created from content at the Biodiversity Heritage Library, the world's largest open access digital library for biodiversity literature and archives. Visit BHL at https://www.biodiversitylibrary.org. 